MiCHEL DEZA

VIATCHESLAV GRISHUKHIN

\title{
Rank 1 forms, closed zones and laminae
}

Journal de Théorie des Nombres de Bordeaux, tome 14, $\mathrm{n}^{\circ} 1$ (2002), p. $103-112$

<http://www.numdam.org/item?id=JTNB_2002_14_1_103_0>

(C) Université Bordeaux 1, 2002, tous droits réservés.

L'accès aux archives de la revue «Journal de Théorie des Nombres de Bordeaux » (http://jtnb.cedram.org/) implique l'accord avec les conditions générales d'utilisation (http://www.numdam.org/conditions). Toute utilisation commerciale ou impression systématique est constitutive d'une infraction pénale. Toute copie ou impression de ce fichier doit contenir la présente mention de copyright.

\section{Numdam}

Article numérisé dans le cadre du programme

Numérisation de documents anciens mathématiques

http://www.numdam.org/ 


\title{
Rank 1 forms, closed zones and laminae
}

\author{
par Michel DEZA et ViatcheSLAV GRISHUKHIN
}

\begin{abstract}
RÉSUMÉ. Étant donné un réseau, nous construisons une équivalence entre des zônes fermées du polytope de Voronoï correspondant, des sections hyperplanes convenables de la partition de Delaunay, et des formes quadratiques de rang 1 qui sont des rayons extrêmes pour les domaines de type $L$ correspondants.
\end{abstract}

\begin{abstract}
For a given lattice, we establish an equivalence between closed zones for the corresponding Voronoi polytope, suitable hyperplane sections of the corresponding Delaunay partition, and rank 1 quadratic forms which are extreme rays for the corresponding $L$-type domain.
\end{abstract}

In this paper we prove a theorem that establishes an equivalence between several notions that play an important role in the theory of quadratic forms and the corresponding lattices. For a quadratic form $f$ and the corresponding lattice $L(f)$, this theorem links:

1) a rank 1 extreme ray of the $L$-type domain to that the form $f$ belongs;

2) a closed zone of parallel edges of the Voronoi polytope $P_{V}$ of $L(f)$;

3) a family of parallel laminar planes of the Delaunay tiling of $L(f)$ which do not intersect Delaunay polytopes of the tiling.

We prove that if the lattice $L(f)$ has one of these properties, then it has all three and any extension of $L(f)$ along edges of the closed zone does not change its $L$-type.

G.Voronoi defined in $§ \S 85-86$ of his famous last paper [6] a domain of quadratic forms of the same $L$-type (or an $L$-type domain) as a set of forms having a given system of positive regulators. In a sense, a regulator characterizes "length" of edges of a zone of the Voronoi polytope $P_{V}$. When a regulator goes to zero, then some edges of the corresponding zone vanish and two Delaunay polytopes glue into one Delaunay polytope. In other words, the Delaunay tiling changes.

According to the above correspondence of a closed zone to a rank 1 extreme ray, the contraction of a closed zone to an open zone relates to a 
transition from an $L$-type domain with a rank 1 form as an extreme ray to one of its faces not containing this ray of rank 1 as an extreme ray.

In his work [2], B. Delaunay used the method of projection along zones for to obtain a classification of four-dimensional Voronoi polytopes.

The partition into Delaunay polytopes is one of two normal partitions of the $n$ space $\mathbf{R}^{n}$ into polytopes that an $n$-dimensional lattice determines. The other is the Voronoi partition. These partitions are dual, i.e. a $k$ dimensional face of one partition is orthogonal to an $(n-k)$-dimensional face of the other partition. Besides, a vertex of one partition is the center of a polytope of the other partition.

The Voronoi partition consists of Voronoi polytopes with its centers in lattice points. Moreover, any polytope of the Voronoi partition is obtained by a translation of the Voronoi polytope with the center in the origin (i.e. the zero lattice point). Call this polytope the Voronoi polytope. It consists of those points of $\mathbf{R}^{n}$ that are at least as closed to 0 as to any other lattice point.

The Delaunay partition consists of Delaunay polytopes which are, in general, not congruent. The set of all Delaunay polytopes having 0 as a vertex is called the star of Delaunay polytopes. Each Delaunay polytope is the convex hull of all lattice points lying on an empty sphere. This sphere is called empty, since no lattice point is an interior point of the sphere.

The Voronoi polytope and the Delaunay polytopes of the star are tightly related to minimal vectors of cosets $2 L$ in $L$. A coset $Q$ is called simple if it contains, up to sign, only one minimal vector.

For a Delaunay polytope $P_{D}$, the lattice vector between any two vertices of $P_{D}$ is a minimal vector of a coset of $L / 2 L$. A lattice vector is an edge of a Delaunay polytope of the star (and then, by duality, it defines a facet of the Voronoi polytope) if and only if it is the minimal vector of a simple coset of $L / 2 L$. All minimal vectors of a non-simple coset are diagonals of a centrally symmetric face of a Delaunay polytope of the star.

The set $\mathcal{P}(P)$ of all faces of all dimensions of a polytope $P$ is partially ordered by inclusion. Call it face poset of $P$. If we reverse the order of $\mathcal{P}\left(P_{V}\right)$, we obtain the poset of those faces of Delaunay polytopes of the star that contain the point 0 . The face poset of the Voronoi polytope $P_{V}$ of a lattice $L$ determines uniquely the combinatorial structure of $P_{V}$ and the $L$-type of the Voronoi and Delaunay partitions. One says that a lattice $L$ belongs to or is of an $L$-type if its Voronoi and Delaunay partitions have this $L$-type. In other words, two lattices (and their Voronoi and Delaunay partitions) belong to the same $L$-type if the corresponding partitions are combinatorially and topologically equivalent. The affine structure of the Delaunay tiling determines uniquely its combinatorial structure. In fact, if two lattices have affinely equivalent Delaunay partitions, then there are 
affinely equivalent bases of these lattices such that the integer coordinates of vertices of equivalent Delaunay polytopes are the same.

There is a small perturbation of a basis of $L$ such that the corresponding form does not go out of its $L$-type domain (this perturbation is only scaling if the dimension of the $L$-type is one. Suppose that a perturbation of the basis changes the $L$-type. Then the Delaunay partition changes, some Delaunay polytopes glue. This means that the spheres of glued Delaunay polytopes coincide. In other words, there is an empty sphere such that a lattice point either leaves or comes onto the sphere.

There is the following simple but important test of emptiness of a sphere. Let $S \subset \mathbf{R}^{n}$ be an $(n-1)$-dimensional sphere with the origin point 0 on it. Let $v_{1}, v_{2}, \ldots, v_{n}$ be $n$ linearly independent vectors with endpoints on $S$. Let $u \in \mathbf{R}^{n}$ be an arbitrary vector and $u=\sum_{i=1}^{n} z_{i} v_{i}$. (We denote by $(p, q)$ the scalar product of vectors $p$ and $q$, and set $\left.p^{2}=(p, p)\right)$.

Lemma 1. (Proposition 4 of [1]). The endpoint of $u$ is not an interior point of $S$ if and only if the following inequality holds

$$
u^{2} \geq \sum_{i=1}^{n} z_{i} v_{i}^{2}
$$

The endpoint of $u$ lies on $S$ if and only if equality holds in (1).

Proof. Let $c$ be the center of $S$. Since the endpoint of $v_{i}$ lies on $S$, we have $\left(v_{i}-c\right)^{2}=c^{2}, 1 \leq i \leq n$, i.e. $v_{i}^{2}=2\left(v_{i}, c\right)$. Multiplying this equality by $z_{i}$, summing over $i$ and taking in attention that $u=\sum z_{i} v_{i}$, we obtain

$$
\sum_{i=1}^{n} z_{i} v_{i}^{2}=2(u, c) .
$$

Since the endpoint of $u$ is not an interior point of $S,(u-c)^{2} \geq c^{2}$, i.e. $u^{2} \geq 2(u, c)$. Using the above equality, we obtain (1). It is easy to see that if equality holds in (1) if and only if the inequality $u^{2} \geq 2(u, c)$ holds as equality, i.e. if and only if the endpoint of $u$ lies on $S$.

Any basis $\mathcal{B}=\left\{b_{i}, 1 \leq i \leq n\right\}$ of an $n$-dimensional lattice $L$ determines uniquely a positive definite quadratic form

$$
f(x)=\left(\sum_{1}^{n} b_{i} x_{i}\right)^{2}=\sum_{1 \leq i, j \leq n} a_{i j} x_{i} x_{j}
$$

The symmetric matrix $a_{i j}$ of the coefficients of this form is the Gram matrix of the basis $\mathcal{B}$, i.e. $a_{i j}=\left(b_{i}, b_{j}\right)$. The matrix $a_{i j}$ can be considered as a point of an $N$-dimensional space, where $N=\left(\begin{array}{c}n+1 \\ 2\end{array}\right)$. In this space, all positive definite forms form an open cone. The closure of this cone is the cone $\mathcal{P}_{n}$ of all positive semi-definite quadratic forms of order $n$. 
One says that a quadratic form belongs to or is of an $L$-type if its lattice belongs to this $L$-type. Hence the cone $\mathcal{P}_{n}$ is partitioned into $L$-type domains of forms (i.e. sub-cones of the cone $\mathcal{P}_{n}$ ) of the same $L$-type. Of course, the cone $\mathcal{P}_{n}$ has many domains of the same $L$-type corresponding to distinct choices of a basis. Voronoi proved that each $L$-type domain is an open polyhedral cone of dimension $k, 1 \leq k \leq N$. An $N$-dimensional $L$-type domain is called general. Domains of other dimensions are called special. Any face of the closure of a general $L$-type domain is the closure of a special $L$-type domain. One-dimensional $L$-type domains are extreme rays of the closure of a general $L$-type domain.

Call a form an edge form if it belongs to a one-dimensional $L$-type domain. In [1], an edge form is called rigid form, since the only transformation of the corresponding lattice that does not change its $L$-type is a scaling. A typical edge form is the square of a linear form: $f(x)=\left(\sum_{i=1}^{n} p_{i} x_{i}\right)^{2}$, i.e. a rank 1 form, where the rank of the form is the rank of its matrix of coefficients. Note that there are no edge forms of rank 2 or 3. But, for $n \geq 4$, there are edge forms also of rank $k \leq n$, for every $k \geq 4$.

A polyhedral domain of quadratic forms is called dicing domain if all extreme rays of its closure are forms of rank 1 with the vectors $p=\left\{p_{i}\right.$ : $1 \leq i \leq n\}$ defining a dicing. Dicings were defined and studied by Erdahl and Ryshkov [4]. In [4], they give conditions when a dicing domain is an $L$-type domain and prove the following theorem (Theorem 4.3 of [4]): $A n$ $L$-type domain is a dicing domain if and only if all the edge forms are rank 1 forms.

Return to the Voronoi polytope $P_{V}$ of an $n$-dimensional lattice $L$. The Voronoi polytope $P_{V}$ itself and its facets (i.e. faces of dimension $n-1$ ) are centrally symmetric, as well as, of course, its vertices and edges. But faces of other dimensions are, in general, not centrally symmetric.

The set of edges of $P_{V}$ is partitioned into classes of mutually parallel edges. These classes are called zones. There are two types of zones: closed and open. A zone is called closed if every two-dimensional face contains either two edges of the zone or none. Otherwise, the zone is called open. Although B.Delaunay used the notion of a closed zone, the explicit definitions of closed and open zones was introduced only by P.Engel in [3].

A closed zone has the following property. Let $l$ be the minimal length of edges of a closed zone $Z$. Let us shorten all edges of $Z$ onto a value $\varepsilon \leq l$. If $\varepsilon<l$, then $Z$ remains closed, and the new polytope $P_{V}^{\prime}$ (with shortened edges) is a Voronoi polytope with the same face poset as $P_{V}$. If $\varepsilon=l$, then $Z$ transforms into an open zone, and $P_{V}^{\prime}$ has another face poset, since at least one edge vanishes.

Since the Voronoi partition is dual to the Delaunay partition, each edge of a Voronoi polytope is orthogonal to a facet of a Delaunay polytope. A facet 
$F$ of a Delaunay polytope of a lattice $L$ generates an affine hyperplane $H$ in $\mathbf{R}^{n}$, namely the hyperplane, where $F$ lies. Obviously, $F$ contains $n$ affinely independent lattice points. Hence the intersection $L \cap H$ is an $(n-1)$ dimensional sub-lattice of $L$. The Delaunay partition of $L$ generates a partition of the hyperplane $H$ into Delaunay polytopes of the lattice $L \cap H$. It may be that all $(n-1)$-dimensional Delaunay polytopes of the partition of $H$ are facets of Delaunay polytopes of the original Delaunay partition of $L$. In this case $H$ is called a lamina of the lattice $L$.

The notion of lamina was introduced and extensively used by Ryshkov and Baranovskii in [5] (see §9.4). If $L$ belongs to a general $L$-type and if a hyperplane $H$ is not a lamina, then it intersects in an interior point an edge of at least one Delaunay polytope $P_{D}$ of the star (Lemma 9.3 of [5]). In other words, there are two vertices of $P_{D}$ that lie in distinct half-spaces determined by $H$. We reformulate Lemma 9.3 of [5] for a lattice of an arbitrary $L$-type.

Lemma 2. A hyperplane is a lamina if and only if it does not intersect any Delaunay polytope of the star in an interior point.

Obviously, a lamina determines a family of parallel laminae that partitions the lattice $L$ into parallel layers, each of them spanning a lamina. Lemma 2 implies the following corollary.

Corollary. Every Delaunay polytope of a lattice with a lamina lies between two neighboring laminae with vertices on these two laminae.

The main property of this partition of $L$ into layers, spanning laminae, is that the distances between layers may be changed without changing the $L$-type of $L$.

Let $H$ be a hyperplane spanning an $(n-1)$-dimensional sub-lattice of $L$. Let $e$ be a unit vector orthogonal to $H$. Then we can define an $\epsilon$-extension along $e$ of the space and of the lattice $L$ as follows. Any vector $v \in \mathbf{R}^{n}$ is uniquely decomposed as $v=v_{e}+v_{H}$, where $v_{e}=(e, v) e$ and $v_{H}$ are the projections of $v$ onto $e$ and $H$, respectively. An $\epsilon$-extension of $\mathbf{R}^{n}$ along $e$ transforms every vector $v$ into the vector

$$
v^{\prime}=(1+\epsilon) v_{e}+v_{H}=\epsilon(e, v) e+v .
$$

Here the $\epsilon$-extension is, in fact, a contraction if $\epsilon<0$. In particular, for the norm (= squared length) $v^{\prime 2}$ of the extended vector, we obtain the following expression, where we set $\lambda=\epsilon(2+\epsilon)$ :

$$
v^{\prime 2}=v^{2}+\lambda(e, v)^{2} .
$$

Of course, an $\epsilon$-extension of $\mathbf{R}^{n}$ along $e$ transforms a lattice $L \subset \mathbf{R}^{n}$ into an extended lattice $L^{\epsilon}$.

There is the following relation between the above introduced notions of a rank 1 form, a closed zone, a lamina and the extended lattice. 
Theorem 1. Let $L$ be an $n$-dimensional lattice, $H$ be a hyperplane spanning an $(n-1)$-dimensional sub-lattice of $L, e$ be an $n$-dimensional unit vector orthogonal to $H$. Let $f(x)$ be the quadratic form corresponding to $a$ basis $\left\{b_{i}: 1 \leq i \leq n\right\}$ of $L$ and $D(f)$ be the $L$-type domain of $f$. The following assertions are equivalent:

(i) $H$ is a lamina of the Delaunay partition of $L$;

(ii) the Voronoi polytope $P_{V}$ of $L$ has a closed zone $Z_{e}$ of edges parallel to the vector $e$;

(iii) the $\epsilon$-extended along e lattice $L^{\epsilon}$ has the same $L$-type as $L$ for all $\epsilon>0$;

(iv) the rank 1 form $f_{e}(x)=\left(e, \sum_{1}^{n} b_{i} x_{i}\right)^{2}$ lies on an extreme ray of the closure of $D(f)$, i.e. $f+\lambda f_{e} \in D(f)$ for all nonnegative $\lambda$.

Proof. (i) $\Rightarrow$ (ii). Let $H$ be a lamina partitioned into facets of Delaunay polytopes of $L$. We can suppose that $H$ contains the origin 0 . Consider the edges of the Voronoi polytope $P_{V}$ that are orthogonal to the lying in $H$ facets of the star. Obviously these edges are parallel to $e$ and form the zone $Z_{e}$. We show that $Z_{e}$ is closed. If not, there is a 2-face $T$ of $P_{V}$ containing exactly one edge $u_{1} \in Z_{e}$. The edges of $T$ form a polygon. Let $u_{1}, u_{2}, \ldots, u_{k}$ be consecutive edges of this polygon. Let $F_{i}$ be the facet of the star that is orthogonal to the edge $u_{i}, 1 \leq i \leq k$. The set of facets $\left\{F_{i}: 1 \leq i \leq k\right\}$ has a common $(n-2)$-dimensional face of the star that is orthogonal to $T$ and lies in the lamina $H$. Since, for $2 \leq i \leq k$, the edge $u_{i}$ is not parallel to $u_{1}$, the facet $F_{i}$ does not lie in the lamina $H$. Hence there is an index $j$ such that the facets $F_{j}$ and $F_{j+1}$ lie in distinct half-spaces separated by $H$. Obviously, $F_{j}$ and $F_{j+1}$ are facets of a same Delaunay polytope $P_{j}$ of the star, and $H$ intersects $P_{j}$. This contradicts to definition of a lamina. Hence $Z_{e}$ cannot be open.

(ii) $\Rightarrow(i)$. Let $u_{1} \in Z_{e}$. Consider the facet $F_{1}$ of the star that is orthogonal to $u_{1}$ and contains $0 \in L$. $F_{1}$ spans a hyperplane $H$ that is orthogonal to $e$ and contains $0 \in L$. Let $T_{1}$ be a 2 -face of $P_{V}$ containing $u_{1}$, and $u_{2}$ be the second edge from $Z_{e}$ contained in $T_{1}$. Let $F_{2}$ be the facet of the star that is dual to $u_{2} . F_{2}$ intersects $F_{1}$ by an $(n-2)$-face that is dual to $T_{1}$ and contains 0 . Hence $F_{2}$ contains 0 and is orthogonal to $e$. This implies that $F_{2}$ lies in $H$. Similarly, for $i=3,4, \ldots$, we consider the 2 -face $T_{i}$ containing $u_{i}, u_{i+1} \in Z_{p}$, and prove that the facet $F_{i}$ of the star dual to $u_{i}$ lies in $H$. Since $P_{V}$ is a polytope, it has a finite number of edges. Hence there is $i_{0}$ such that $u_{i_{0}}=u_{1}$. We obtain a set of facets of the star lying in $H$ such that the facet $F_{i}$ intersects $F_{i-1}$ and $F_{i+1}$. Therefore the intersection of the star with $H$ consists of facets of the star. Since this is true for all Voronoi polytopes having centers in $H$, the hyperplane $H$ is partitioned into facets of Delaunay polytopes. This means that $H$ is lamina. 
(i) $\Rightarrow$ (iii). It is sufficient to prove that no lattice point comes onto or leaves the empty sphere $S$ of a Delaunay polytope $P_{D}$. Without loss of generality, we can suppose that $P_{D}$ belongs to the star. Let $v_{1}, v_{2}, \ldots, v_{n}$ be $n$ linearly independent lattice vectors with endpoint in vertices of $P_{D}$, i.e. they lie on the sphere $S$. Let $u$ be any lattice vector and let $u=\sum_{i=1}^{n} z_{i} v_{i}$ be its decomposition by $v_{i}, 1 \leq i \leq n$. According to (2), after an $\epsilon$-extension of the space along $e, u$ and $v_{i}$ are transformed into the vectors

$$
u^{\prime}=u+\epsilon(u, e) e, v_{i}^{\prime}=v_{i}+\epsilon\left(v_{i}, e\right) e .
$$

By Lemma 1 , it is sufficient to prove that the inequality ${u^{\prime 2}}^{2} \geq \sum_{i=1}^{n} z_{i} \boldsymbol{v}_{i}^{\prime 2}$ is strict or is an equality according to the inequality $u^{2} \geq \sum_{i=1}^{n} z_{i} v_{i}^{2}$ is strict or is an equality.

Recall that $H$ contains an $(n-1)$-dimensional sub-lattice. Hence $(v, e)=$ $k(v) \alpha$ for any lattice vector $v$, where $k(v)$ is an integer and $\alpha$ does not depend on $v$. Let $k=k(u)$ and $k_{i}=k\left(v_{i}\right)$. Multiplying the equality $u=\sum_{i=1}^{n} z_{i} v_{i}$ by $e$, we obtain the equality $k=\sum_{i=1}^{n} z_{i} k_{i}$. By Corollary of Lemma 2, the vertices of $P_{D}$ lie on two neighboring laminae. Hence $\left|k_{i}\right|=0,1$. Without loss of generality we can suppose that $k_{i} \geq 0$, i.e. $k_{i}=0,1$ and therefore $\left(v_{i}, e\right)^{2}=k_{i}^{2} \alpha^{2}=k_{i} \alpha^{2}$. Hence, using (3), we have

$$
{v_{i}^{\prime}}^{2}=v_{i}^{2}+\lambda\left(v_{i}, e\right)^{2}=v_{i}^{2}+\lambda k_{i} \alpha^{2} .
$$

Since $\sum_{i=1}^{n} z_{i} k_{i}=k$, we obtain the equality

$$
\sum_{i=1}^{n} z_{i}{v_{i}^{\prime}}^{2}=\sum_{i=1}^{n} z_{i} v_{i}^{2}+\lambda \alpha^{2} \sum_{i=1}^{n} z_{i} k_{i}=\sum_{i=1}^{n} z_{i} v_{i}^{2}+\lambda \alpha^{2} k .
$$

Suppose that $u^{2}>\sum_{i=1}^{n} z_{i} v_{i}^{2}$. We show that then $\sum_{1}^{n} z_{i} v_{i}^{\prime 2}<{u^{\prime}}^{2}=u^{2}+$ $\lambda(u, e)^{2}=u^{2}+\lambda \alpha^{2} k^{2}$. For an integer $k$, we have $k \leq k^{2}$ with equality if and only if $k=0,1$. The above inequalities and the equality (4) imply

$$
\sum_{1}^{n} z_{i} v_{i}^{\prime 2}<u^{2}+\lambda \alpha^{2} k^{2}=u^{\prime 2}
$$

Now let $u^{2}=\sum_{1}^{n} z_{i} v_{i}^{2}$. This means that $u$ has the endpoint in a vertex of $P_{D}$. Hence $k=0,1$, i.e. $k^{2}=k$. Hence the equality (4) implies the equality

$$
\sum_{1}^{n} z_{i} v_{i}^{2}=u^{2}+\lambda \alpha^{2} k^{2}=u^{\prime 2}
$$

(iii) $\Rightarrow$ (iv). We prove in the implication (i) $\Rightarrow$ (iii) that $f^{\epsilon}:=f\left(L^{\epsilon}\right) \in D(f)$ for every $\epsilon \geq 0$. Now we show that

$$
f\left(L^{\epsilon}\right)=f(L)+\lambda f_{e},
$$


where $\lambda=\epsilon(2+\epsilon)$. The basic vectors of the extended lattice $L^{\epsilon}$ have the form

$$
b_{i}^{\prime}=b_{i}+\epsilon\left(e, b_{i}\right) e, \quad 1 \leq i \leq n .
$$

Hence the coefficients $a_{i j}^{\prime}$ of the quadratic form $f^{\epsilon}=f\left(L^{\epsilon}\right)$ are as follows

$$
a_{i j}^{\prime}=\left(b_{i}^{\prime}, b_{j}^{\prime}\right)=\left(b_{i}, b_{j}\right)+\lambda\left(e, b_{i}\right)\left(e, b_{j}\right) .
$$

Hence we obtain

$$
f^{\epsilon}(x)=f(x)+\lambda\left(\sum_{i=1}^{n}\left(e, b_{i}\right) x_{i}\right)^{2}=f(x)+\lambda f_{e}(x) .
$$

This means that the ray $\left\{\lambda f_{e}: \lambda \geq 0\right\}$ belongs to the closure $\operatorname{cl} D(f)$ of $D(f)$. Since this ray is a one-dimensional $L$-type domain, it is an extreme ray of $\operatorname{cl} D(f)$.

(iv) $\Rightarrow$ (iii). If $f_{e}$ lies on an extreme ray of $\operatorname{cl} D(f)$, then the quadratic function $f^{\epsilon}=f+\epsilon(2+\epsilon) f_{e}$ belongs to $D(f)$ for all $\epsilon \geq 0$. The matrix $a_{i j}^{\prime}$ of $f^{\epsilon}$ is

$$
a_{i j}+\epsilon(2+\epsilon)\left(e, b_{i}\right)\left(e, b_{j}\right)=\left(b_{i}^{\prime}, b_{j}^{\prime}\right) .
$$

Hence $f^{\epsilon}$ is a quadratic form of the $\epsilon$-extended lattice $L^{\epsilon}$. So, $L^{\epsilon}$ has the same $L$-type as $L$ for all $\epsilon \geq 0$.

(iii) $\Rightarrow$ (i). We show that if the extended along $e$ lattice $L^{\epsilon}$ has the same $L$-type as $L$ for all $\epsilon>0$, then the hyperplane $H$ which is orthogonal to $e$ is a lamina. Suppose $H$ is not a lamina. Then $H$ intersects a Delaunay polytope $P_{D}$ of the star in an interior point. Hence there are two vectors $v_{1}$ and $v_{2}$ with endpoints in vertices of $P_{D}$ such that $k_{1}=k\left(v_{1}\right) \geq 1$ and $k_{2}=k\left(v_{2}\right) \leq-1$. Consider the lattice vector

$$
u=q\left(k_{1} v_{2}-k_{2} v_{1}\right)
$$

where the integer $q$ is chosen such that the endpoint of $u$ does not lie on the empty sphere $S$ circumscribing $P_{D}$. Expand the pair of vectors $v_{1}, v_{2}$ up to a set of $n$ independent vectors with endpoints in vertices of $P_{D}$. Then the above expression for $u$ is the representation of $u$ as a linear combination of these $n$ independent vectors. Since the endpoint of $u$ does not lie on $S$, by Lemma $1, \Delta:=u^{2}-q\left(k_{1} v_{2}^{2}-k_{2} v_{1}^{2}\right)>0$.

Consider an $\epsilon$-extension of the space along $e$. Let $u^{\prime}, v_{1}^{\prime}, v_{2}^{\prime}$ be the $\epsilon$ extended vectors. Consider the difference $\Delta^{\prime}:={u^{\prime}}^{2}-q\left(k_{1} v_{2}^{\prime 2}-k_{2} v_{1}^{\prime 2}\right)$. Using (3), we obtain

$$
\Delta^{\prime}=\Delta+\lambda(e, u)^{2}-q\left(k_{1} \lambda\left(e, v_{2}\right)^{2}-k_{2} \lambda\left(e, v_{1}\right)^{2}\right) .
$$

Since $(e, u)=\alpha k(u)=0,\left(e, v_{1}\right)=\alpha k_{1},\left(e, v_{2}\right)=\alpha k_{2}$ and $k_{2}<0$, we have

$$
\Delta^{\prime}=\Delta-\lambda \alpha^{2} q k_{1}\left|k_{2}\right|\left(\left|k_{2}\right|+k_{1}\right) .
$$


Let $p=\alpha^{2} q k_{1}\left|k_{2}\right|\left(k_{1}+\left|k_{2}\right|\right)>0$. Then for $\lambda=\frac{\Delta}{p}$, we obtain $\Delta^{\prime}=0$. Lemma 1 implies that, for this $\lambda$, the endpoint of $u$ lies on $S$. This means that there is $\epsilon>0$ such that the $\epsilon$-extended lattice $L^{\epsilon}$ has the $L$-type distinct from the $L$-type of $L$. We obtain a contradiction.

Remark 1. The rank 1 function $f_{e}(x)=\left(e, \sum_{1}^{n} b_{i} x_{i}\right)^{2}$ depends on the basis corresponding to the function $f(x)$. But this dependence is not essential in the following sense. Recall that $\left(e, b_{i}\right)=\alpha k\left(b_{i}\right)$, where $k_{i}=k\left(b_{i}\right)$ is an integer. If we slightly move $f(x)$ in the domain $D(f)$, then we slightly change the basis $\mathcal{B}=\left\{b_{i}: 1 \leq i \leq n\right\}$ and the scalar products $\left(e, b_{i}\right)=\alpha k_{i}$. But, since $k_{i}$ is an integer, it cannot change slightly. Hence only $\alpha$ slightly changes. This implies that this movement of $f(x)$ inside of the $L$-type domain $D(f)$ causes a movement of $f_{e}(x)=\alpha^{2}\left(\sum_{1}^{n} k_{i} x_{i}\right)^{2}$ along the ray $\left\{\lambda\left(\sum_{1}^{n} k_{i} z_{i}\right)^{2}: \lambda \geq 0\right\}$. The collections of the integers $\left\{k_{i}: 1 \leq i \leq n\right\}$ is an invariant of the $L$-type domain $D(f)$.

Remark 2. The equivalence (i) $\Leftrightarrow$ (iv) of Theorem 1 is mentioned in the paper [4]. After the proof of Theorem 4.3, the authors of [4] write:

The ideas used in this proof can be extended to cover the case in which only a portion of the edge forms have rank 1 . For such an $L$-type domain each rank 1 edge form can be associated with a $D$ family of parallel hyperplanes $G$, and the $L$-partitions $\mathcal{S}$ of lattices on this domain are refinement of the partition determined by $G$. In the other direction, any hyperplane which does not intersect the interior of any $L$-polytope of an $L$-partition can be associated with a rank 1 edge form of the corresponding $L$-type domain. Such hyperplanes are members of a $D$-family of parallel hyperplanes.

Note that here an $L$-partition and an $L$-polytope mean a Delaunay partition and a Delaunay polytope, and a $D$-family is a family of parallel laminae.

In fact, the authors of [4] asserts that the ideas used in the proof of their Theorem 4.3 can be extended to the proof of the equivalence (i) $\Leftrightarrow$ (iv). But our proof of this equivalence is different from one suggested in [4].

\section{References}

[1] E. P.BaranovskiI, V. P.Grishukhin, Non-rigidity degree of a lattice and rigid lattices, European J. Combin. 22 (2001), 921-935.

[2] B. N. Delaunay (Delone), Sur la partition régulière de l'espace à 4 dimensions. Izvestia AN SSSR ser. matem. 1 (1929), 79-110 et 2 (1929), 145-164.

[3] P. ENGEL, Investigations of parallelohedra in $\mathbf{R}^{d}$. In: P.Engel, H.Syta eds., Voronoi's impact on modern science, Institute of Mathematics, Kyiv 1998, vol. 2, 22-60.

[4] R. M. ERdahl, S. S. Ryshkov, On lattice dicing. European J. Combin. 15 (1994), 459-481. 
[5] S. S. RYShKov, E. P. BARANovskII, C-types of n-dimensional lattices and 5-dimensional primitive parallelohedra (with application to the theory of covering). Trudy of Steklov's Mathematical Institute, vol. 137 (1976), 3-131. (Translated as: Proceedings of Steklov Institute of Mathematics 1978, No 4.)

[6] G. F. VoronoI, Nouvelles applications des paramètres continus à la théorie des formes quadratiques - Deuxième mémoire. J. Reine Angew. Math. 134 (1908), 198-287 et 136 (1909), 67-178.

Michel DezA

École Normale Supérieure

Paris, France

E-mail : Michel.DezaCens.fr
Viatcheslav GRISHUKHIN

CEMI, Russian Academy of Sciences

Moscow, Russia

E-mail : grishuhnecemi.rssi.tu 